\title{
LM-I-25
}

\section{Dual wavelength double fs-pulse laser irradiation for fused silica processing}

\author{
$\underline{\text { J. Lopez }}^{1}$, K. Gaudfrin ${ }^{1,2}$, K. Mishchik ${ }^{3}$, M. Delaigue ${ }^{3}$, C. Hoenninger ${ }^{3}$, E. Audouard ${ }^{3}$, L. \\ Gemini $^{2}$, R. Kling ${ }^{2}$, G. Duchateau ${ }^{1}$ \\ 1- Univ.Bordeaux, CNRS, CEA, CELIA UMR 5107, 33405 Talence, France \\ 2- Alphanov, 33405 Talence, France \\ 3- Amplitude Systemes, 33600 Pessac, France \\ john.lopez@u-bordeaux.fr
}

Ultrafast laser glass processing is a subject of high interest for many industrial applications in optics, medical device, or micro electronics industries. Indeed, ultrafast laser technology has the unique capacity to produce a high-quality surface or bulk modification in dielectric transparent materials thanks to nonlinear absorption. However, the throughput achieved with ultrafast lasers is still low compared to other laser technologies, laser-assisted chemical etching or mechanical processing. Temporal or spatial beam shaping is a smart and flexible technique to overcome this limitation and to meet industry requirements.

In the present work, we investigate the benefits and the drawbacks in using on dual-wavelength double fs-pulse laser irradiation for fused silica processing. Our purpose of this pump-pump experiment is to tune the electron dynamics in order to optimize energy deposition and then to improve ablated volume. We use green wavelength $(515 \mathrm{~nm})$ for the first pulse to enhance photo-ionization and near-infrared $(1030 \mathrm{~nm})$ for the second pulse to maximize electron heating and impact ionization. The investigated parameters are pulse-to-pulse delay (up to $20 \mathrm{ps}$ ), second pulse duration ( 1 and $10 \mathrm{ps)}$ ) and total fluence (up to $20 \mathrm{~J} / \mathrm{cm}^{2}$ ). The results will be discussed in terms of ablated volume and optical transmission. We demonstrate that (i) there is an optimum delay and (ii) the ablation behavior is intermediate between green and near-infrared single pulse irradiation. Our results are supported by a numerical model taking into account electron dynamics and absorbed energy density. 